\title{
LE DRESSEUR D'OURS DE LA BORDURE INFÉRIEURE DE LA BRODERIE DE BAYEUX: POUR UNE NOUVELLE INTERPRÉTATION*
}

\author{
Xavier Barral i Altet
}

\author{
X. Barral i Altet \\ University of Rennes II-Haute Bretagne, France \\ Università di Venezia Ca'Foscari, Italy \\ email: xavierbarralaltet33@gmail.com
}

\begin{abstract}
The Bayeux Embroidery portrays the invasion of England by William the Conqueror, who defeated Harold's English army at the battle of Hastings in 1066. Its rich content, depicts a collective imaginary of the Middle Ages, and displays a major source of information in order to visualize daily life in the $11^{\text {th }}$ and first half of the $12^{\text {th }}$ centuries. The whole tissue is framed by two borders showing fantastical animals, monsters or fables, such as the one of the crow and the fox, and combatants, participating within the broader story. In this article the author tries to identify a scene in the lower border: a man training and showing a bear.
\end{abstract}

Key words: Bayeux, embroidery, training animals, bear, borders, iconography

La broderie de Bayeux illustre l'histoire de la conquête de la couronne d'Angleterre par le duc de Normandie, Guillaume, et s'achève par la bataille de Hastings qui eut lieu le 14 octobre 1066. À travers la narration de cet épisode historique précis, la broderie nous présente de nombreux détails de vie quotidienne de la société contemporaine $e^{1}$. Le récit se déroule de gauche à droite avec quelques retours. Les stratégies de représentation sont nombreuses et variées, souvent inspirées très directement de l'Antiquité ${ }^{2}$. La manière de déployer l'histoire appartient à un genre narratif qui devait orner les parties hautes de la salle de réception d'un palais, comme les frises peintes qui, au cours du Moyen Âge et aux époques successives, ornaient le haut des murs de ce type d'espaces solennels, en rapport souvent avec le décor peint du plafond.

Dans la broderie de Bayeux on trouve trois récits parallèles imbriqués mais autonomes. Il s'agit de trois manières d'expliquer une histoire qui est délimitée en haut et en bas par deux lignes continues: la frise centrale, les inscriptions et les bordures. Les correspondances profondes entres ces trois récits nous échappent souvent. Le récit central, principal, occupe le milieu de la bande et est le plus large. Le récit des inscriptions est une vraie histoire racontée avec des mots qui illustrent et élargissent ce

\footnotetext{
${ }^{1}$ Une bibliographie, déjà très abondante en 1988, avait été réunie par S. A. BROWN, The Bayeux Tapestry. History and Bibliography, Woolbridge, 1988. Depuis, elle l'avait actualisée : S. A. BROWN, La tapisserie de Bayeux : analyse critique des publications des années 1988-1999, in : P. BOUET, B. LEVY, F. NEVEUX (eds.), La Tapisserie de Bayeux: l'art de broder l'Histoire. Actes du colloque de Cerisy-la-Salle (1999), Caen, 2004, pp. 27-47. En 2013, un nouveau volume a été consacré à la bibliographie de la broderie : S. A. BROWN, The Bayeux Tapestry. Bayeux, Médiathèque municipale: Ms. 1. A Sourcebook, Turnhout, 2013. On trouvera d'excellentes photographies dans les nouvelles éditions de D. M. WILSON, La Tapisserie de Bayeux, Paris, 2005, et celle de L. MUSSET, The Bayeux Tapestry, Woolbridge, 2005. Parmi les références collectives les plus récentes: La tapisserie de Bayeux, l'art de broder l'histoire, actes du colloque de Cerisy-la-Salle, 1999, Caen, 2004 ; M.K. FOYS, K.E. OVERBEY, D. TERKLA (eds.), The Bayeux Tapestry. New Interpretations, Woolbridge, 2009 ; M. J. LEWIS, G. R. OWEN-CROCKER, D. TERKLA (eds.), The Bayeux Tapestry. New approaches. Proceedings of a conference at the British Museum, Oxford, 2011. Voir également le recueil d'écrits de G. R. OWEN-CROCKER, The Bayeux Tapestry. Collected papers, Farnham, 2012.
} 
que l'on voit dans les images avec leur propre autonomie, comme le texte d'une bande dessinée sans images. Enfin, le récit des bordures est double et se situe en haut et en bas de la bande principale avec une succession d'épisodes, parfois interrompue lorsque les images du secteur principal débordent sur les bordures, que l'on a eu parfois des difficultés à expliquer par rapport au déroulement des scènes majeures. Ces bordures ont une très grande cohérence entre elles et apportent le complément nécessaire au récit principal, même si nous avons parfois des difficultés à mettre en relation les images des bordures avec celles qui leur correspondent dans la bande principale. Qu'il y a un rapport étroit et une seule unité de l'histoire le prouvent les débordements et les empiètements d'un territoire sur l'autre et les endroits où, de toute évidence, les récits apparaissent complémentaires ${ }^{3}$.

Les bordures de la broderie de Bayeux présentent, dans leurs parties conservées, des animaux, des êtres hybrides, des petites scènes de vie quotidienne, soigneusement alignées et séparées par des éléments végétaux ou géométriques. Parfois les épisodes de la frise centrale débordent sur les bordures ${ }^{4}$. En général, le thème de la nature est omniprésent dans les bordures de la broderie, comme dans l'art médiéval en général ${ }^{5}$. Les animaux inscrits dans les bordures extérieures accompagnent les scènes sur toute la longueur de l'ouvrage ${ }^{6}$. Les animaux y prennent donc une grande place $e^{7}$. Ils sont présentés par couples, deux bêtes affrontées ou adossées, isolés ou dans le cadre de scènes. Il s'agit d'animaux domestiques ou sauvages et d'animaux monstrueux, des animaux familiers et des animaux exotiques. Parmi les premiers, nous reconnaissons des chiens, des béliers, des chats, des paons, des canards, des grues, des lions ou des dromadaires, entre autres. Parmi les seconds, nous observons des hommes et des femmes centaures, des chevaux ailés, des monstres à queue ou à tête nouée, des créatures chimériques etc. La figure du lion est l'un des motifs les plus récurrents dans les bordures de la broderie de Bayeux et, de façon plus générale, dans l'iconographie romane occidentale.

${ }^{2}$ O. K. WERCKMEISTER, The Political Ideology of the Bayeux Tapissery, in Studi medievali, 17 (1976), pp. 535-595, réimprimé dans Romanesque Art and Ideology. Reprinted Articles, Los Angeles, 1978, pp. 119-192 ; G. R. OWEN-CROCKER, Stylistic Variation and Roman Influence in the Bayeux Tapestry, in Peregrinations, 2 (2009), 4, pp. 51-96 ; A. PELLEGRINELLI, La narrazione figurata di Bayeux e la tradizione classica, Udine, 2007 ; X. BARRAL I ALTET, Observations sur l'organisation narrative de la broderie de Bayeux et ses rapports avec l'Antiquité, in Les Cahiers de Saint-Michel de Cuxa, 39 (2008), pp. 31-46.

${ }^{3}$ Je traite globalement de ces questions dans un ouvrage sous presse à paraitre prochainement aux Editions du Cerf, à Paris.

${ }^{4}$ Sur les bordures de la broderie, la bibliographie est également abondante. Voir, particulièrement, J. ABRAHAM, A. LETIENNE, Les bordures de la Tapisserie-Broderie de Bayeux, in Normania, 2 (1929), pp. 483-518 ; D. J. BERNSTEIN, The Mistery of the Baveux Tapestry, Londres, 1986, pp. 124-135 ; A. KUHN, Der Teppich von Baveux in seinen Gebärden: Versuch einer Deutung, in Studi medievali, XXXIII (1992), pp. 1-72; X. MURATOVA, Ideologia dei margini e margini dell'ideologia. Riflessioni sui margini dell'Arazzo di Bayeux e sui programmi delle zone secondarie nella decorazione dei monumenti medievali, in: Medioevo: immagini e ideologia. Atti del convegno (Parma, 2002), Milano, 2005, pp. 657-670 ; M. JULLIAN, Les bordures de la Tapisserie de Bayeux : un ornement?, in Revue d'Auvergne, 124 (2010), pp. 89-114 ; P. KLEIN, The borders of the Bayeux Tapestry: visual gloss or marginal images?, in : Le plaisir de l'art du Moyen Age. Mélanges en hommage à Xavier Barral i Altet, Paris, 2012, pp. 626-642.

${ }^{5}$ R. LENOBLE, Histoire de l'idée de nature, Paris, 1969; B. KIILERICH, The Abundance of Nature - The Wealth of Man: Reflections on an Early Byzantine Seasons Mosaic from Syria, in : E. PILTZ, P. ASTRÖM (ed.), Kairos. Studies in Honour of G. Akerström-Hougen, Jonsered, 1998, pp. 22-36 ; X. MURATOVA, Guardare la natura, in : E. CASTELNUOVO, G. SERGI (eds.), Arti e storia nel Medioevo, III, Turin, 2004, pp. 437-472; M. VAN DER LUGT (ed.), La nature comme source de la morale au Moyen Âge, Florence, 2014 (Micrologus' Library, 58).

${ }^{6}$ B. W. YAPP, Animals in Medieval Art: The Bayeux Tapestry as an Example, in Journal of Medieval History, XIII (1987), pp. 15-73 ; C. HICKS, The Animals on the Borders of the Bayeux Tapestry, in C. HICKS (ed.), England in the Eleventh Century. Proceedings of the 1989 Harlaxton Symposium, Stamford, 1992, pp. 251-265.

${ }^{7}$ F. D. KLINGENDER, Animals in Art and Thought to the End of the Middle Ages, Londres, 1971 ; L'uomo di fronte al mondo animale nell'Alto Medioevo, Centro Italiano di Studi sull'Alto Medioevo, Spoleto, 1985 (Settimane di Studio, 31); C. HICKS, Animals in Early Medieval Art, Edinburgh, 1993, particulierement pp. 252-270 ; A. PARAVICINI BAGLIANI (ed.), Il mondo animale. The World of Animals, Florence, 2000 (Micrologus' Library, 8, 1-2). 
Il y a également des groupes d'animaux qui interviennent dans les représentations de fables, bien connues depuis l'Antiquité, comme le corbeau et le renard, le loup et l'agneau ou le loup et la grue 8 . Dans ce domaine, le livre classique de Léon Hermann ${ }^{9}$ a surinterprété la présence de fables dans les bordures en cherchant a tout prix à mettre en relation toutes les images des bordures avec des fables d'Esope ${ }^{10}$.

C'est sur l'une de ces images que je voudrais m'arrêter particulièrement ici et offrir ces réflexions a l'ami Igor Fisković en souvenir de notre vieille amitié et de tant de promenades dans les paysages de Croatie, avant et après l'indépendance de son pays.

La scène objet de ces réflexions se trouve placée dans la bordure inferieure, sous la représentation de deux messagers de Guillaume à cheval qui galopent de droite à gauche vers Guy

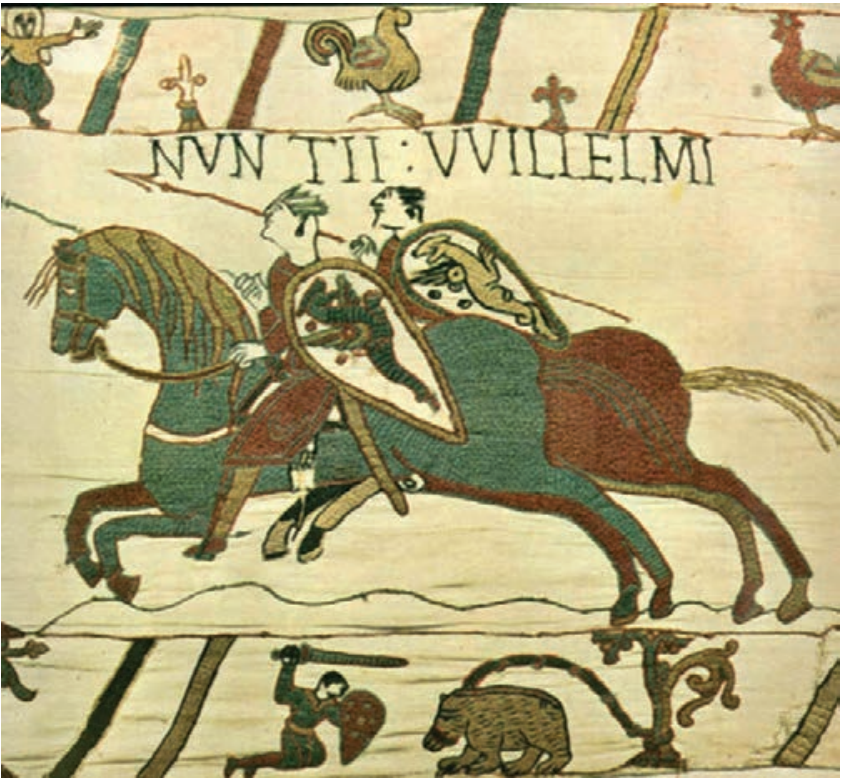

Fig. 1 - Bayeux, Broderie de Bayeux, Dresseur d'ours. de Ponthieu identifiés par l'inscription Nuntii Willelmi (fig. 1). Encadrée par deux séparations formées chacune de deux bandes obliques qui l'isolent d'un épisode animalier et d'une scène de chasse, notre scène occupe une place bien visible et centrée dans la bordure inferieure. Un homme, vêtu d'une tunique verte, courte, ceinte a la taille sur d'épais bas marrons est muni d'un écu en forme d'amande qu'il tient de la main gauche et d'une forte épée qu'il arbore de la droite. L'épée est énorme, théâtrale, comme si elle était en bois, et ne ressemble pas à celles que portent les guerriers sur la frise centrale. Le bouclier est orné d'un point jaune central et de cinq points verts disposés en cercle, comme des clous décoratifs en métal. Devant lui, un ours brun (ursus arctos), le plus connu dans l'hémisphère septentriona ${ }^{11}$, énorme mais fort paisible, est attaché à un arbre très ornemental. La large corde nouée à la base de l'arbre lui ceint le museau mais n'est pas tendue. L'animal ne montre aucune agressivité et l'homme, presque à genoux, semble dialoguer, gesticulant, avec l'animal. Celui-ci est à l'écoute. A la ligne de sol de couleur gris verte qui donne unité à la scène se superpose une ligne ocre rouge qui définit le territoire de l'animal entre l'arbre et les pattes de l'ours.

Les premiers historiens de la broderie n'ont pas porté attention à cette scène, comme d'ailleurs en général à celles des bordures. En revanche, Léon Herrmann, qui cherchait à tout prix à faire coïncider chaque scène des bordures avec une fable antique, proposa une interprétation de cet épisode qui a

\footnotetext{
${ }^{8}$ Les références bibliographiques sur les fables de la broderie de Bayeux sont nombreuses Parmi les publications les plus récentes : J. RUBIO TOVAR, Las fábulas del tapiz de Bayeux, in Revista de poética medieval, 11 (2003), pp. 93125 ; A. BRIDGEFORD, 1066. The Hidden History of the Bayeux Tapestry, Londres-New York, 2004, pp. 66-97 ; P. K. KLEIN, The Meaning of the Fables in the Bayeux Tapestry, in : J. M. LUXFORD, M. A. MICHAEL (eds.), Tributes to Nigel Morgan. Contexts of Medieval Art: Images, Objects \& Ideas, London-Turnhout, 2010, pp. 335-347. Voir les références bibliographiques indiquées par S. A. BROWN, The Bayeux Tapestry, cit., 2013, p. 310.

9 Les recueils les plus utilisés sont ceux de H. CHEFNEUX, Les Fables dans la Tapisserie de Bayeux, in Romania, 60 (1934), n. 237-238, pp. 1-35, pp. 153-194 ; L. HERRMANN, Les fables antiques de la broderie de Bayeux, Bruxelles, 1964 (Collection Latomus, 69).

${ }^{10}$ Ce qui a souvent été dénoncé : par exemple, M. PARISSE, La tapisserie de Bayeux. Un documentaire du XIe siècle, Paris, 1983, p. 132 ; D. M. WILSON, La Tapisserie de Bayeux, cit., p.177, ou L. MUSSET, The Bayeux Tapestry, cit., p. 80.

${ }^{11}$ M. COUTURIER, L'ours brun : ursus arctos, Grenoble, 1954 ; J.-J. CAMARRA, J. RIBAL, L'ours brun, Paris, 1989 ; C. BECK, Approches des territoires de l'ours en Europe au Moyen Âge, in : Actes du XVI colloque de la Société française pour l'étude et la protection de mammifères, Muséum d'Histoire naturelle de Grenoble, Grenoble, 1993, pp. 94-100.
} 


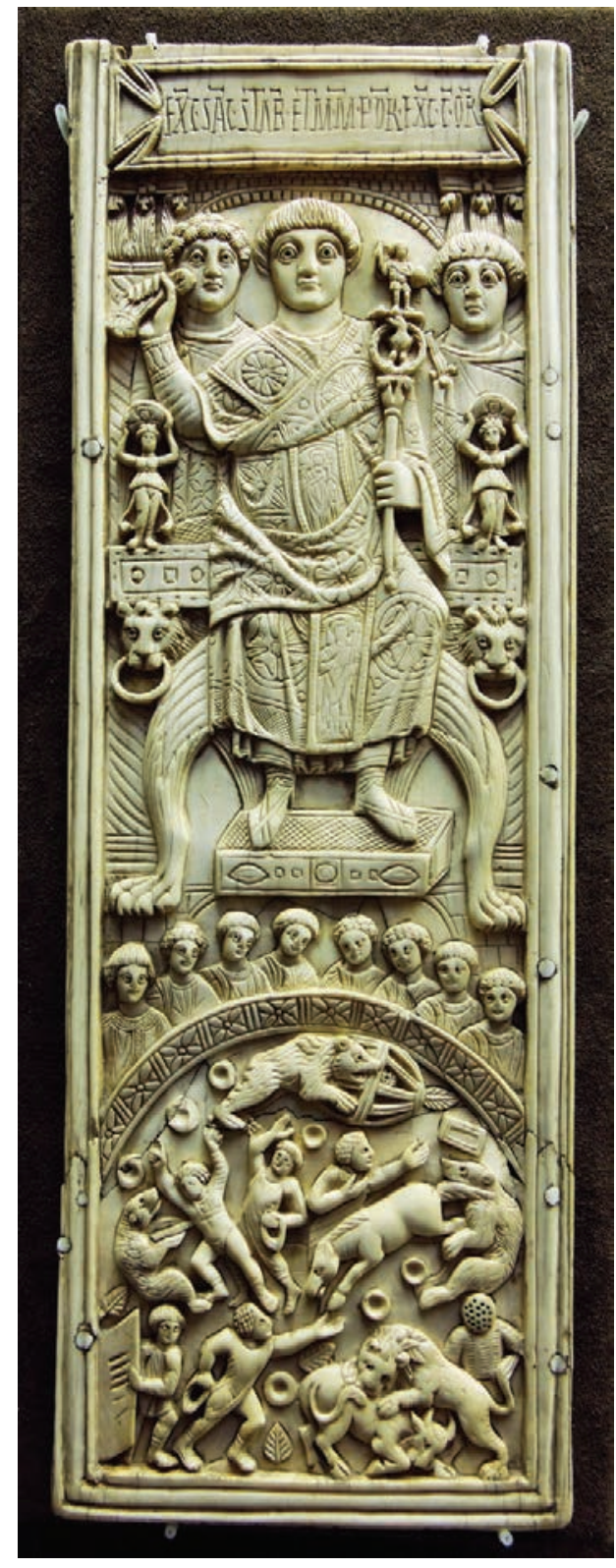

Fig. 2 - Paris, Musée national du Moyen $\hat{A} g e$, Feuillet de diptyque consulaire d'Areobindus (VI siècle), Ours dans l'amphithéâtre. connu une certaine postérité : "Je vais proposer une identification paradoxale. Il s'agit, selon moi, de la fable Le cheval et le sanglier. Et pourtant nous ne voyons pas de cheval, mais un cavalier, et pas de sanglier, mais un ours avec une laisse. Mais le guerrier accroupi, portant au bras gauche un bouclier triangulaire et oblong et au bras droit un glaive immense, n'est autre qu'un chevalier qui a mis pied à terre et dégaine pour combattre. D'autre part, il est clair que l'ours ne se trouve là que par adaptation d'une initiale d'un manuscrit représentant un ours en laisse et a remplacé le sanglier plus difficile à représenter. La fable se réfère en réalité à la vengeance du cavalier contre une bête sauvage qui a troublé le breuvage de son cheval ». Puis, citant Phèdre (III, 39 [IV, 4]), Herrmann continuait : "Le gué où le cheval avait coutume d'étancher sa soif fut troublé par un sanglier qui s'y vautra. Un litige en naquit. L'animal au sabot sonore, irrité contre le fauve, demanda secours a l'homme et, l'élevant sur son dos, revint vers son ennemi. Lorsque le cavalier eut tué celui-ci à coups de javelots, on dit qu'il s'exprima ainsi : 'Je suis ravi de t'avoir porté secours comme tu m'en priais, car j'ai pris cette proie et appris quelle est son utilité'. Et c'est ainsi qu'il le força à subir, contre son gré, le mors. Alors l'autre, attristé : 'En cherchant dans ma folie à me venger d'une bagatelle, j'ai trouvé la servitude!'. Cette fable achèvera d'avertir les gens irascibles qu'il vaut mieux se laisser impunément léser que se livrer à autrui » ${ }^{12}$

J'ai voulu transcrire complètement ces deux passages car ils illustrent bien une certaine manière de détourner les images au profit d'une théorie préconçue, prédéterminée. Je n'ai presque pas besoin de contrer les arguments. Si on avait voulu représenter le sanglier les artistes savaient très bien le faire comme le prouvent les nombreuses représentations qui existent depuis l'Antiquité tardive jusqu'à l'époque romane et au-delà, périodes au cours desquelles la chasse au sanglier était très pratiquée ${ }^{13}$. L'ours possède également ses propres caractéristiques spécifiques que les artistes ont su représenter a toutes les époques (fig. 2). Quant à imaginer que pour figurer un cheval on ait utilisé la représentation d'un personnage c'est une question de perception générale de l'art médiéval que je

ne partage pas. Selon moi, l'identification de l'homme avec une figure masculine et de l'animal avec un ours ne font pas de doute. L'envergure du corps de l'animal, son museau, le pelage et les larges pattes permettent de l'identifier sans difficulté.

On a parfois vu dans cette scène la représentation d'un chasseur ou la lutte d'un homme avec un ours. Mais s'il s'agissait d'une scène de chasse, l'animal ne serait pas attaché et il serait figuré en train d'attaquer ou de fuir. Par ailleurs, les représentations d'époque romane que nous connaissons

\footnotetext{
${ }^{12}$ L. HERRMANN, Les fables antiques, cit., p. 8 et 31.

${ }^{13}$ M. PASTOUREAU, Chasser le sanglier. Du gibier royal a la bête impure : histoire d'une dévalorisation, in : IDEM, Une histoire symbolique du Moyen Âge occidental, Paris, 2004, pp. 73-88, pp. 395-400.
} 
de lutte d'un homme avec un ours sont d'une toute autre violence suivant la tradition des venationes des amphithéâtres romains, dont on en a un bel exemple avec le feuillet de diptyque en ivoire dit d'Areobindus, du milieu du VI ${ }^{e}$ siècle, du Musée national du Moyen Âge à Paris ${ }^{14}$ (fig. 2).

L'ours était considéré au Moyen Âge comme le roi des animaux avant que le lion ne vienne prendre sa place ${ }^{15}$. Dans une miniature du début du XIVe siècle d'un manuscrit d'un Speculum humanae salvationis, conservé a la bibliothèque de Kremsmunster en Autriche (codex 242, fol. 19), David s'attaque à la fois à un lion et a un ours, et déchire la mâchoire de ce dernier. L'artiste a parfaitement et clairement distingué l'anatomie et les caractéristiques de chaque animal ${ }^{16}$. Le culte ancestral de l'ours était au Moyen Âge encore très répandu et de nombreux saints l'ont adopté comme symbole dans les légendes hagiographiques et dans l'iconographie, comme saint Corbinien, saint Amand ou saint Arige. L'ours devint le compagnon de saint Colomban et le complice de saint Gall, une complicité souvent illustrée dans l'art médiéval ${ }^{17}$. Une célèbre plaque de reliure en ivoire qui recouvrait un livre d'évangiles du début du X $\mathrm{X}^{\mathrm{e}}$ siècle de la bibliothèque de Saint-Gall (Cod. Sang., 53) montre la présence directe de l'ours dans deux épisodes de la vie du saint ${ }^{18}$. Enfin, dans certaines régions, saint Ours ou Ursin, le saint qui porte le nom de l'animal, fait l'objet d'un culte particulier ${ }^{19}$. Sa force et sa bravoure en ont toujours fait un animal craint, mais aussi mystérieux par son temps d'hibernation l'hiver, et célébré lors de sa sortie des cavernes qui symbolise l'arrivée du printemps. Le 2 février et les fêtes païennes de l'ours christianisé étaient un moment fort du calendrier médiéval, dans lequel l'ours est traditionnellement associé au mois de février ${ }^{20}$.

L'ours était chassé au Moyen Âge, plus pour sa peau que pour sa viande jugée peu comestible en dehors de certains cercles aristocratiques ${ }^{21}$. On croyait également aux vertus médicinales de sa graisse et de sa bile, considérée comme le remède a beaucoup de maux, et on appréciait certaines parties du corps, comme canines, griffes ou poils, que l'on portait pour bénéficier de la force de l'animal. Mais le plus grand exploit était de chasser l'ours vivant, afin de le dompter, de l'apprivoiser et de le montrer dans les foires, sur les places ou dans les cours ${ }^{22}$.

Pendant longtemps j'ai cru que la scène de la bordure inferieure de la broderie de Bayeux représentait un montreur d'ours ${ }^{23}$, un spectacle qui jouissait de grande acceptation pendant le Moyen Âge et les siècles successifs, un prédécesseur des cirques modernes ${ }^{24}$ (fig. 3 ). Je ne le pense plus et je ne crois pas non plus qu'il s'agisse d'une scène de chasse à l'ours car, au Moyen Âge, le plantigrade

${ }^{14}$ J.-P. CAILLET, L'Antiquité classique, le haut Moyen Age et Byzance au Musée de Cluny, Paris, 1985.

${ }^{15}$ M. PASTOUREAU, L'ours. Histoire d'un roi déchu, Paris, Seuil, 2007. Auparavant, toujours indispensable, R. DELORT, Les animaux ont une histoire, Paris, 1984.

${ }^{16}$ M. PASTOUREAU, L'ours, cit., fig. 16.

${ }^{17}$ The Life of St Gall, edited by M. JOYNT, Burnham on Sea, 1927 ; M. SCHÄR, Gallus. Der Heiliger in seiner Zeit, Bale, 2011.

${ }^{18}$ M. PASTOUREAU, L'ours, cit., fig. 20.

${ }^{19}$ N. LE LUEL, Études des vies de saint Ursin de Bourges : une première approche, in Annales de Bretagne et des pays de l'Ouest, 114 (2007), 1, pp. 4-31.

${ }^{20}$ N. LE LUEL, Comment christianiser un ours? Le mois de février du calendrier du portail Saint-Ursin de Bourges (premier quart du XII siècle), in Cahiers de civilisation médiévale, 55 (2012), pp. 161-172.

${ }^{21}$ M. PRANEUF, L'ours et les hommes dans les traditions européennes, Paris, 1989.

${ }^{22}$ F.-R. GASTOU, Sur les traces des montreurs d'ours des Pyrénées et d'ailleurs, Toulouse, 1987 ; J. DESSE, F. AUDOINROUZEAU (eds.), Exploitation des animaux sauvages a travers le temps, XIII Rencontres internationales d'archéologie et d'histoire d'Antibes (15-17 octobre 1992), Juan-les-Pins, 1992. Voir également l'ouvrage général de P. LOEVENBRUCK, Les animaux sauvages dans l'histoire, Paris, 1955.

${ }^{23}$ Voir l'évocation de Ph. LEGENDRE KVATER, Le montreur d'ours, Paris, diverses éditions, 1990-1996.

${ }^{24}$ Lucien Musset avait déjà évoqué cette possibilité, bien qu'il préféra se tourner vers une "scène de 'bear baiting' si apprécié des Anglais jusqu'au XVIe siècle» : L. MUSSET, The Bayeux Tapestry, cit., p. 80. 


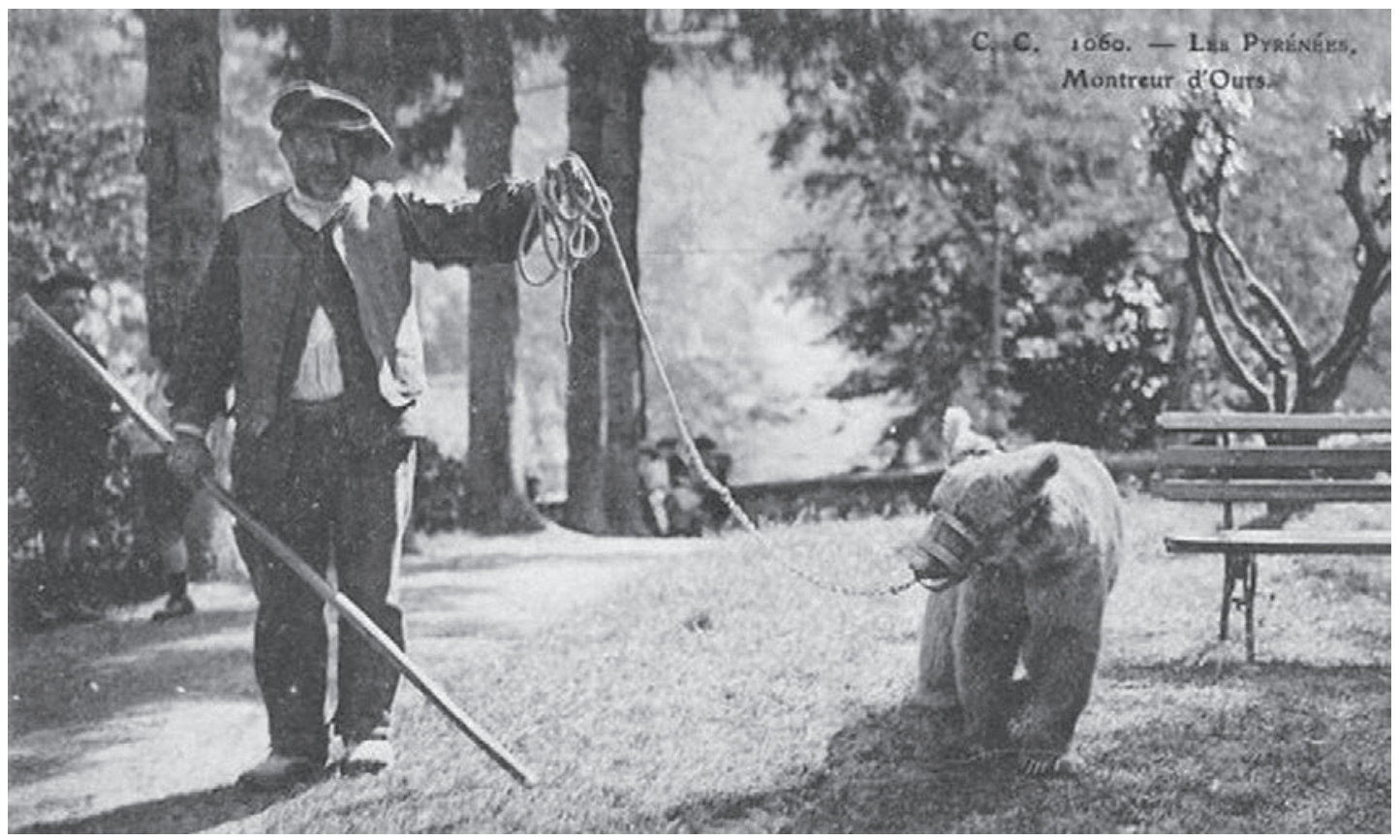

Fig. 3 - Carte postale, Montreur d'ours des Pyrénées au début du XXe siècle.

était habituellement chassé à l'aide de chiens et avec des lances à la pointe élargie en forme de feuille de laurier et dotées d'une petite protubérance cruciforme, comme on le voit par exemple dans une miniature du début du XIVe siècle du Codex Manesse, dans laquelle le chasseur transperce l'ours avec sa lance ${ }^{25}$. Encore moins je m'oriente vers une représentation de "bear baiting ", comme l'avait proposé Lucien Musset ${ }^{26}$. Ce type de harcèlement de l'ours entre chasse et jeu cruel a l'appât, était très violent et d'une toute autre nature que l'atmosphère paisible que respire notre image. L'ours "bêté» que l'on voit dans un décor de marge d'une bible anglaise enluminée vers 1360, conservée a la British Library de Londres (Ms. Harley 4189, fol. 114) montre bien la violence du spectacle et la lutte acharnée entre l'ours et plusieurs chiens ${ }^{27}$. Cette pratique fut très populaire en Angleterre après le Moyen Âge et jusqu'à la fin de l'époque moderne, mais dénoncée par sa cruauté déjà au cours du XVIe siècle ${ }^{28}$ et jusqu'à aujourd'hui ${ }^{29}$. Shakespeare dans ses Merry Wives of Windsor avait immortalisé un ours, le fameux Sackerson du Paris-Garden ${ }^{30}$, le colisée construit près de la Tamise et très fréquenté pour les spectacles d'ours, survivant héroïque d'une de ces versions modernes des spectacles de l'arène antique si prisées et populaires pendant l'ère élisabéthaine ${ }^{31}$.

La scène de Bayeux représente un dresseur d'ours, l'étape préalable et indispensable pour le montreur d'ours. L'homme gesticule et est équipé des instruments nécessaires pour se défendre en cas de

\footnotetext{
${ }^{25}$ M. PASTOUREAU, L'ours, cit., l'a reproduite a la figure 7.

${ }^{26}$ Lucien Musset avait évoqué cette possibilité (voir note 24).

${ }^{27}$ On trouvera cette scène reproduite par M. PASTOUREAU, L'ours, cit., fig. 23.

${ }^{28}$ J. FIELD, A Godly Exhortation, by Occasion of the Late Judgement of God, Shoed in Paris-Garden the Thirteenth Day of January, Londres, 1583 ; R. PREISS, Interiority, in: H. S. TURNER (ed.), Early Modern Theatricality, Oxford, 2013 (Oxford Twenty-First Century Approaches to Literature), p. 52.

${ }^{29}$ J. JOSEPH, Bear-baiting in Pakistan, World Society for the Protection of Animals, Londres, 1997.

${ }^{30}$ Dans l'acte I, scene 1, Slender dit : «I have seen Sackerson loose twenty times, and have taken him by the chain ».

${ }^{31}$ Pour l'Antiquité, parmi une bibliographie assez nourrie : D. BOMGARDNER, The Trade in Wild Beasts for Roman Spectacles : A Green Perspective, in Anthropozoologica, 16 (1992), pp. 161-166 ; D. GOGUEY, Les romains et les animaux: regards sur les grands fauves, dans Homme et Animal dans l'Antiquité romaine, Tours, 1995, pp. 51-66. Et, maintenant, la réédition de l'ouvrage classique de G. VILLE, La Gladiature en Occident des origines à la mort de Domitien (avec une préface de Paul Veyne), Rome, 2014 (Classiques. École française de Rome,.
} 


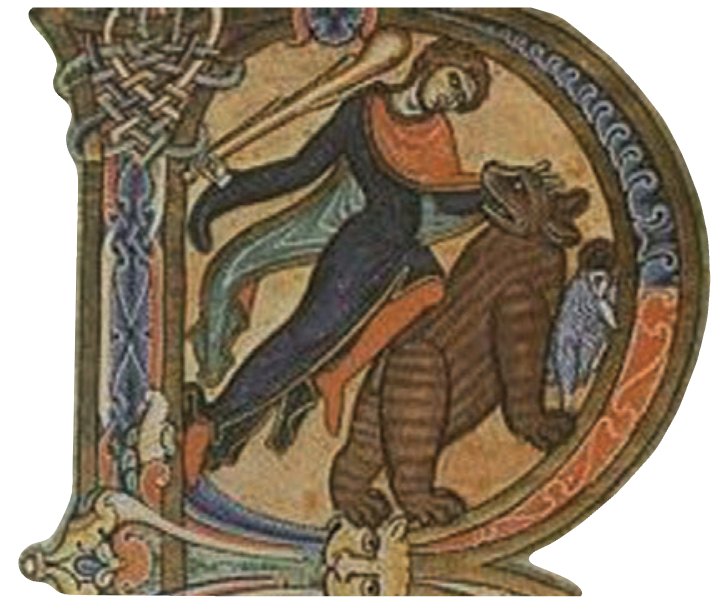

Fig. 4 - Cathédrale de Winchester, Bible de Winchester (1160-1180), Dressage difficile d'un ours.

besoin. L'animal écoute avec attention car il est déjà à un stage avancé du dressage. La corde qui lui ferme la gueule et le tient à la base de l'arbre n'est pas tendue. C'est la corde avec laquelle le dresseur, devenu montreur, tiendra l'ours dans ses futures promenades d'exhibition.

L'iconographie médiévale offre de nombreux exemples de relation entre l'homme et l'ours ${ }^{32}$ et surtout de nombreux épisodes de dressage ainsi que du spectacle ou l'ours est simplement montré à la curiosité et devant la crainte de tous ${ }^{33}$. L'homme se protège avec un bâton et tient l'ours en laisse tandis que celui-ci a la gueule muselée, une image récurrente que l'on trouve encore identique chez les montreurs d'ours à la fin du XIX siècle et pendant le premier tiers du XXe

Deux initiales enluminées, celles de la Bible de Winchester ${ }^{34}$ (fig. 4) et d'un manuscrit des commentaires de saint Augustin sur l'évangile de Jean de la Bibliothèque municipale de Tours (fig. 5) évoquent et nous montrent deux étapes successives de l'apprivoisement de l'animal ; tandis que dans la première, l'homme cherche à dominer l'ours lui posant un genou sur le dos, dans la seconde l'animal suit déjà docilement son maître. Sur un chapiteau roman de la nef de Saint-Pierre-le-Moûtier (Nièvre), l'animal est déjà dressé et s'exhibe debout devant son maître. Une inscription indique clairement qu'il s'agit d'un ours $^{35}$ (fig. 6). Sur une mosaïque romane de l'ancienne cathédrale de Casal Monfer-

\footnotetext{
${ }^{32}$ M. MONTANARI, Uomini e orsi nelle fonti agiografiche dell'alto medioevo, in : G. BLASCHITZ, G. JARITZ (eds.), Symbole des Alltags. Alltag der Symbole. Festschrift für Harry Kühnel, Graz, 1992, p. 571-587; M. SALVAT, L'ours dans la symbolique médiévale, in: A. NIDERST (ed.), L'animalité : hommes et animaux dans la littérature française, Tübingen, 1994, pp. 55-66.

${ }_{33}$ B. ANDREOLLI, L'orso nella cultura nobiliare dall'Historia Augusta a Chrétien de Troyes, in : B. ANDREOLLI, M. MONTANARI (eds.), Il bosco nel Medioevo, Bologne, 1989, pp. 35-54.

${ }^{34}$ C. DONOVAN, The Winchester Bible, Toronto, 1993.

${ }^{35}$ A. GONAT, Notice historique sur Saint-Pierre-le-Moûtier, Nevers, 1908 ; M. ANFRAY, L'architecture religieuse du Nivernais au Moyen Age: les églises romanes, Paris, 1951 ; J. DUPONT, Nivernais-Bourbonnais Roman, La Pierre-quiVire, 1976 ; Ch. SAPIN, C. ARNAUD, W. BERRY, Bourgogne Romane, Dijon, 2006.
} 


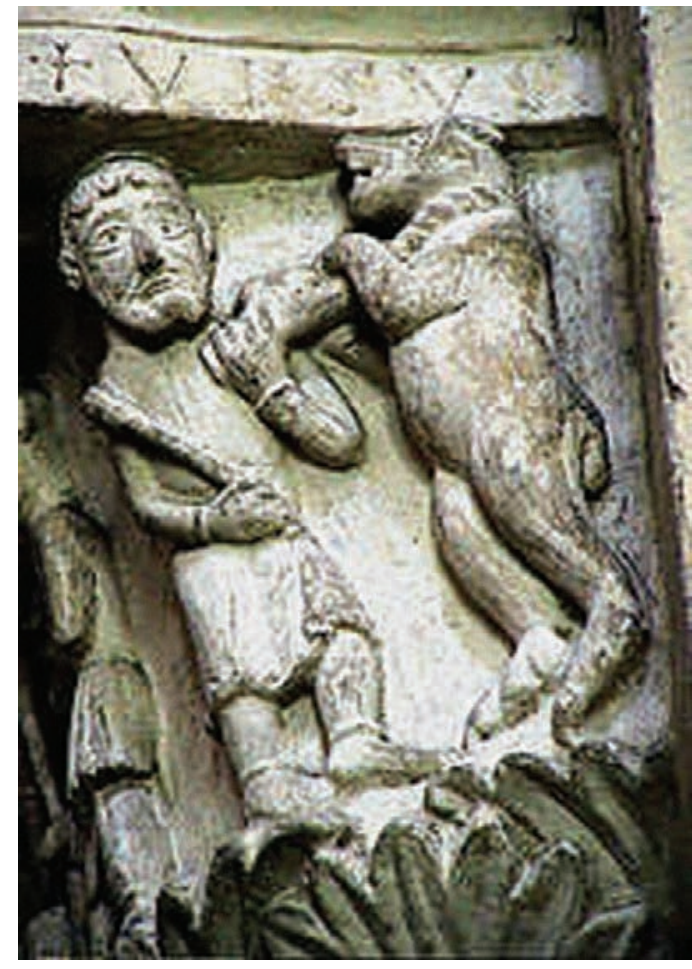

Fig. 6 - Saint-Pierre-le-Moûtier (Nièvre). Chapiteau de la nef, Ours exhibé par le montreur.

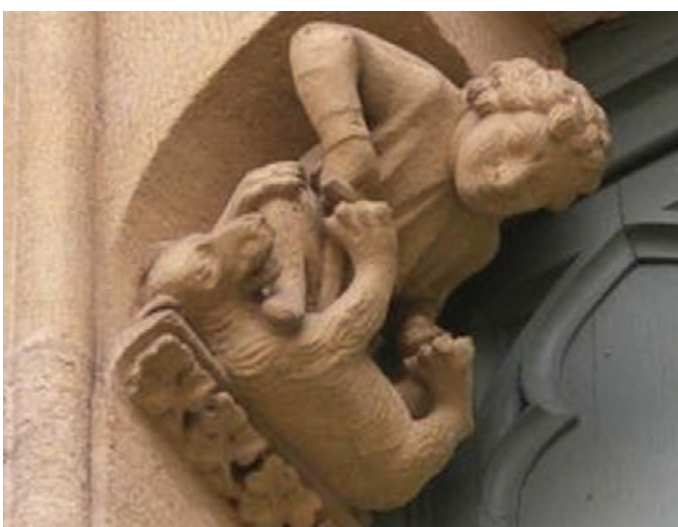

Fig. 8 - Metz, Cathédrale Saint-Etienne, Façade, Dressage difficile d'un ours.

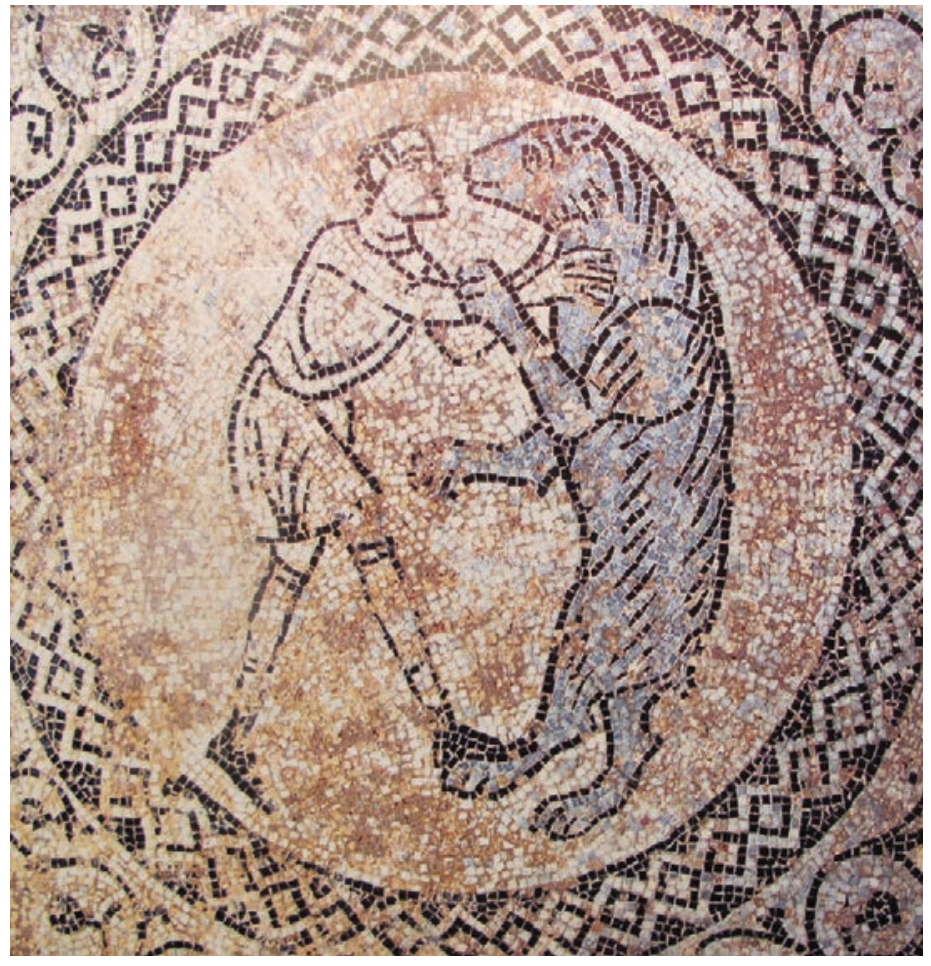

Fig. 7 - Casale Monferrato, Mosaïque de l'ancienne cathédrale, Dressage d'un ours.

rat, dans le Piémont, l'homme dresse l'animal gentiment, sans bâton ni laisse ${ }^{36}$ (fig. 7). Selon moi, dans ce cas, il ne s'agit pas non plus d'une scène de lutte mais de dressage. Le dressage devait avoir ses moments de difficulté et de danger car il devient presque une bataille entre l'homme et l'ours dans la façade de la cathédrale Saint-Etienne de Metz $^{37}$ (fig. 8). Apres le dressage, le montreur d'ours pouvait se produire en spectacle et faire faire à l'ours les acrobaties apprises. On le voit dans la belle enluminure du manuscrit de la vie de saint Denis conservé a la Bibliothèque nationale de France et daté de 1317, dans laquelle un ours guidé par son maitre fait des acrobaties sur un pont de Paris $^{38}$ (fig. 9). À la fin du XVe siècle, une belle

${ }^{36}$ E. CECCHI GATTOLIN, I tessellati romanici a figure del Duomo di San Evasio a Casale Monferrato, in : Scritti di Storia dell'Arte in onore di Roberto Salvini, Florence, 1984, pp. 37-44; X. BARRAL I ALTET, Il mosaico pavimentale del duomo romanico di Casale Monferrato : osservazioni sull'iconografia, in : A. CASAGRANDE, G. PARODI TRAVAGLIA (eds.), Arte e carte nella diocesi di Casale, Alessandria, 2007 (Provincia di Alessandria : i tesori delle sue diocesi, 3), pp. 156173; IDEM, Le décor du pavement au Moven Âge : les mosaïques de France et d'Italie, Rome, 2010 (Collection de l'École française de Rome, 429), p. 311-312.

${ }^{37}$ J.-M. PIERRON, La cathédrale de Metz, Moselle, Nancy, 1994 ; Ch. BRACHMANN, La construction de la cathédrale SaintÉtienne de Metz et de l'église collégiale Notre-Dame-la-Ronde pendant le deuxième tiers du XIIIe siècle, in : Congrès archéologique de France. $149^{\circ}$ session. Les Trois-Evêchés et l'ancien duché de Bar. 1991, Paris, 1995, pp. 447-475 ; A. VILLES, Remarques sur les campagnes de construction de la cathédrale de Metz au XIII siècle, in Bulletin Monumental, 162 (2004), pp. $243-272$.

${ }^{38}$ V. W. EGBERT, On the Bridges of Medieval Paris. A Record of Early Fourteenth Century Life, Princeton, 1974 ; C. LACAZE, The vie de saint Denis manuscript (Paris, Bibliothèque nationale, ms. fr. 2090-2092), New York-Londres, 1979; P. MANE, 


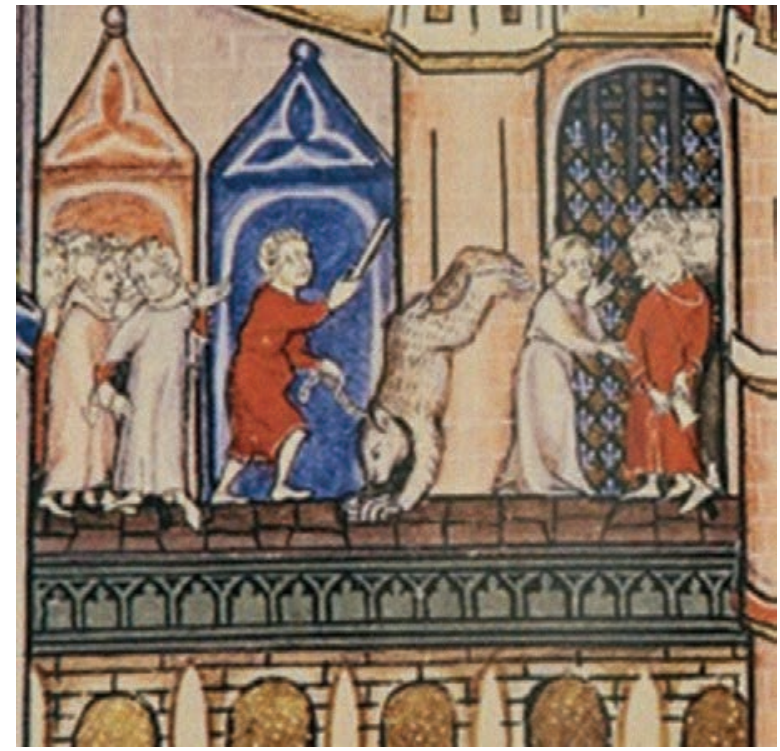

Fig. 9 - Paris, BnF, ms. fr. 20091, fol. 47, Vie de saint Denis (1317), Montreur d'ours sur un pont de Paris.



Fig. 10 - Paris, BnF, ms. fr. 2829, fol.22, Guillaume de Saint-Pathus, Vie et miracles de saint Louis (fin $d u X^{e}$ siècle), Montreur d'ours.

image d'un manuscrit de la vie et des miracles de saint Louis nous montre une scène analogue dans un tout autre contexte (fig. 10).

Les images médiévales de dresseurs et de montreurs d'ours sont nombreuses et témoignent de la présence remarquée et souhaitée de ce spectacle exotique dans la vie quotidienne. Reste à comprendre son sens dans la bordure inferieure de la broderie de Bayeux. Dans les différents contextes médiévaux, qu'il s'agisse d'initiales de manuscrits, de chapiteaux ou de mosaïques, l'épisode apparait toujours isolé au sein de cycles ou de séries d'images de nature très différente. Elles sont, comme à Bayeux, parfois religieuses ${ }^{39}$, parfois séculières ${ }^{40}$. L'image de Bayeux évoque un fait habituel dans la vie des villes, des campagnes et des cours du Moyen Âge, mais qui était tout de même exceptionnel par sa nature au sein de la quotidiennetét ${ }^{4}$.

Ce sont ces aspects anecdotiques qui donnent à la broderie de Bayeux toute sa personnalité médiévale ${ }^{42}$. Le programme général cache encore beaucoup d'inconnues ${ }^{43}$. Dans ce contexte, je n'ose développer une hypothèse hasardeuse sans davantage de preuves. Je pense cependant que la scène de l'ours dans la bordure de Bayeux est une évocation plus ou moins directe du mois de février. Le mois de février est utilisé dans certains calendriers romans pour commencer le cycle de l'année comme sur le tympan de l'église Saint-Ursin de Bourges ${ }^{44}$. Notons au passage que sur le tympan de Saint-

Métiers et artisanats urbains : images et réalité, in : M. BOONE, E. LECUPPRE-DESJARDIN, J.-P. SOSSON (eds.), Le verbe, l'image et les représentations de la société urbaine au Moyen Âge. Actes du colloque international (Marche-en-Famenne, 24-27 octobre 2001), Anvers/Apeldoorn, 2002, pp. 109-118, en part. pp. 115-117.

39 J. BERLIOZ, M.-A. POLO DE BEAULIEU (eds.), L'animal exemplaire au Moyen Âge (Ve-XVé siècle), Rennes, 1999.

${ }^{40}$ C. R. DODWELL, A Brief Note on the Secular Aspects of the Bayeux Tapestry, in Gazette des Beaux-Arts, LXVIII (1966), pp. 227-232; IDEM, The Bayeux Tapestry and the French Secular Epic, in The Burlington Magazine, CVIII (1966), pp. $549-560$

${ }^{41}$ Sur la présence d'animaux sauvages ou rares pour le plaisir individuel ou collectif : G. LOISEL, Histoire des ménageries de l'Antiquité à nos jours, Paris, 1912.

${ }^{42}$ D. ALEXANDRE-BIDON, M.-TH. LORCIN, Le quotidien au temps des Fabliaux : textes, images, objets, Paris, 2003. Sur le contexte littéraire, M. CAILLY, Les fabliaux, la satire et son public. L'oralité dans la poésie satirique et profane en France, XII ${ }^{e}$-XIVE siècles, Cahors, 2007.

${ }^{43}$ M. PASTOUREAU, La broderie de Bayeux: un programme introuvable?, in : J.-M. GUILLOUËT, C. RAVEL, (eds.), Le programme : une notion pertinente en Histoire de l'art médiéval ?, Paris, 2011 (Cahiers du Léopard d'Or, 12), 95-134.

${ }^{44}$ Sur cette question, N. LE LUEL, Comment christianiser un ours?, cit. 


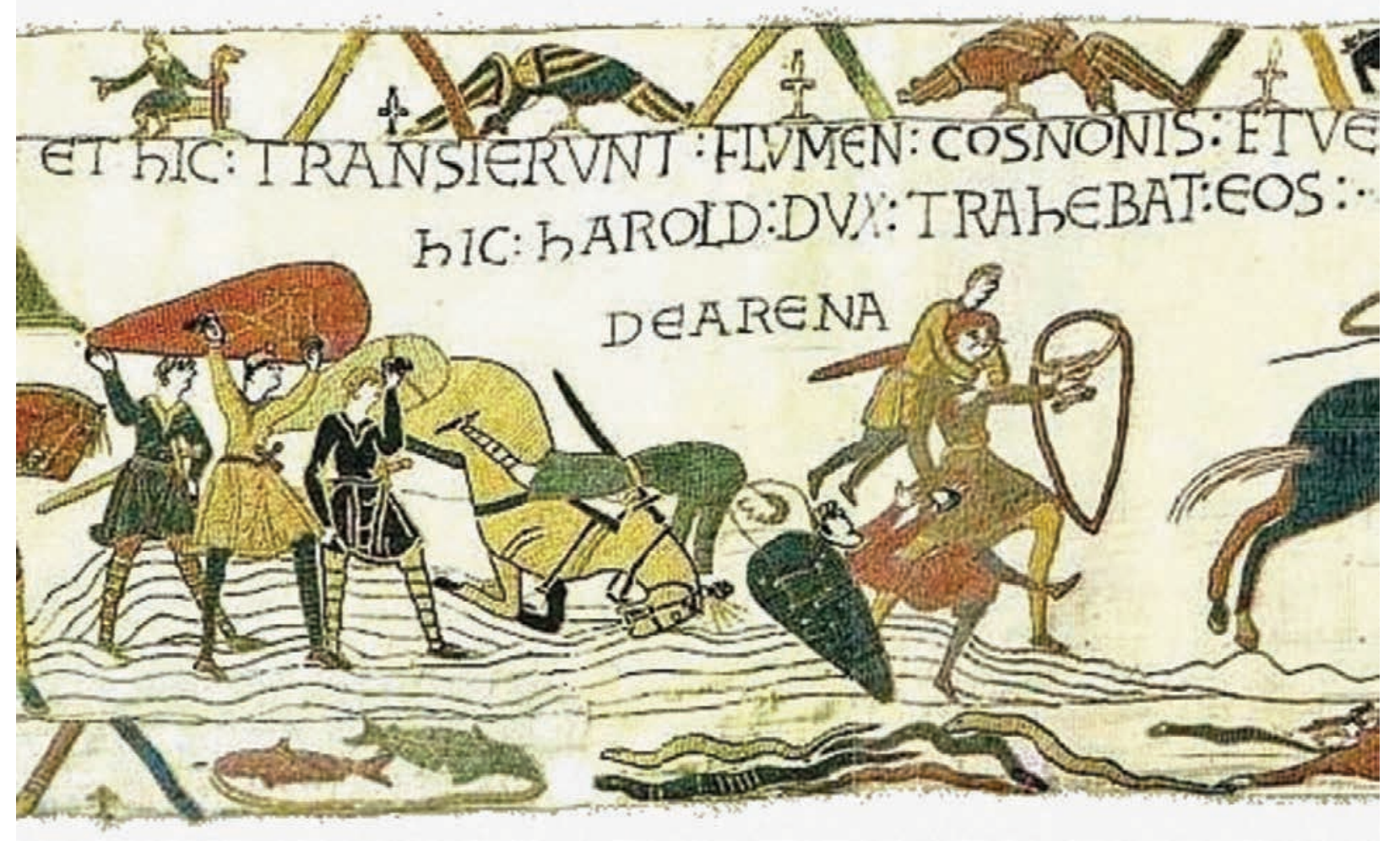

Fig. 11 - Bayeux, Broderie de Bayeux, Signe zodiacal des Poissons.

Ursin de Bourges, le cycle des mois accompagne un frise de chasse et une série de fables, comme à Bayeux $^{45}$. Dans le récit de la bordure inferieure de la broderie de Bayeux apparaissent, un peu plus tôt, des scènes de travaux des champs, qui pourraient cacher une autre référence au cycle des mois dans ce cas probablement les laboureurs du mois de mars ${ }^{46}$.

Un peu plus loin dans la même bordure, sous l'épisode des sables mouvants lors de la traversée du Couesnon, deux poissons sont présentés en opposition de couleur, rouge et verte, et de direction, mais clairement liés entre eux par une ligne continue, une sorte de cordon filiforme jaune cerné de rouge qui les relie par la bouche (fig. 11). Ils évoquent le Zodiaque à travers le signe astrologique des Poissons qui correspond à la deuxième partie du mois de février et à la première du mois de mars. Ce signe symbolise dans les Zodiaques médiévaux la fin de l'hiver et de la ronde des signes et est généralement associé à l'élément classique de l'eau. Le signe des Poissons est toujours représenté de cette manière, sous la forme de deux poissons jumeaux nageant en sens contraire, comme on le voit souvent au Moyen Âge, par exemple dans le vitrail de la cathédrale de Chartres ou, plus tôt, dans le Zodiaque de la mosaïque de pavement de la cathédrale d'Otrante, dans les Pouilles, ou il accompagne la personnification du mois de mars ${ }^{47}$. Le cordon reliant les deux poissons apparait souvent, comme dans le portail Saint-Firmin de la façade occidentale de la cathédrale d'Amiens. Ce détail distingue, dans la bordure inferieure de la broderie de Bayeux, les poissons du signe zodiacal des simples poissons

\footnotetext{
${ }^{45} \mathrm{~N}$. LE LUEL, L'âne, le loup, la grue et le renard : à propos de la frise des fables du tympan Saint-Ursin de Bourges, in Reinardus, 18 (2005), pp. 53-68.

${ }^{46}$ Certains calendriers médiévaux commençaient par le mois de mars : ibidem, p. 64, note 16, et P. MANE, Calendriers et techniques agricoles (France-Italie, XII et XIII siècles), Paris, 1983, p. 46 ; G. COMET, Les calendriers médiévaux. Une représentation du monde, in Journal des savants, (1992), 1, pp. 35-97 (en part. pp. 46-47) ; M. A. CASTIÑEIRAS, El calendario medieval hispano. Textos e imágenes (siglos XI-XIV), Valladolid, 1996.

${ }^{47}$ G. GIANFREDA, I mesi nel mosaico di Otranto: oroscopo o tempo di Dio e lavoro dell'uomo?, Lecce, 2004; X. BARRAL I ALTET, Le décor du pavement, cit., p. 364-370.
} 
figurés plus loin dans l'histoire, peu après l'apparition de la comète. Les signes du Zodiaque sont très souvent représentés, comme on sait, à l'époque romane, sur toutes formes de supports et particulièrement fréquents sur les pavements en mosaïque romans. ${ }^{48}$

Pour encadrer une confrontation humaine, celle entre l'insulaire Harold et le Normand Guillaume et pour situer géographiquement les épisodes de guerre, les villes, les fortifications, les églises et les palais, le concepteur du programme iconographique de la broderie de Bayeux a envisagé des bordures. Celles-ci entourent l'histoire principale, l'enfermant complètement. A droite, la bordure qui devait clore le cycle a été perdue avec les dernières scènes de l'histoire. Mais ici les bordures servent à encercler l'histoire principale en nous proposant une vision du monde, avec les animaux, les signes du Zodiaque, le temps de l'année, les scènes de la vie rurale ou les fables animalières.

Le monde physique de la frise principale, réel et habité, est cerné d'un monde imaginaire, concentré plus spécifiquement dans les bordures, dans lequel chaque image évoque un aspect concret, connu ou supposé. La continuité linéaire des épisodes des bordures de la broderie de Bayeux me semble se situer à l'opposé de la logique qui sous-tend les illustrations marginales des manuscrits, avec lesquelles on a souvent voulu rapprocher les images des bordures de Bayeux ${ }^{49}$. En aucun cas les marginalia des manuscrits ne possèdent une continuité narrative sur l'ensemble du manuscrit ${ }^{50}$; elles ne correspondent pas à la logique d'une bordure continue comme on les trouve dans les peintures murales et surtout les pavements depuis l'Antiquité ${ }^{1}$. Dans ce cas il s'agit d'un réel encadrement ${ }^{52}$. Dans les bordures de Bayeux on trouve les éléments, les saisons et les mois, le ciel, la terre et l'eau, les animaux en tant que notations géographiques ${ }^{53}$ et des épisodes dont chacun évoque un aspect précis de la vie quotidienne. Puisque la totalité du monde habité ne pouvait pas y être figuré on avait choisi des évocations qui à l'époque étaient évidentes mais que nous, faute de documents écrits, penons encore à expliquer totalement.

\footnotetext{
${ }^{48} \mathrm{Ph}$. CALDER NYE, The Romanesque Signs of the Zodiac, in Art Bulletin, V (1922-1923), pp. 55-57; M. VIEILLARDTROIEKOUROFF, Les zodiaques parisiens sculptés d'après Le Gentil de la Galaisère, astronome du XVIII siècle, in Mémoires de la Société nationale des Antiquaires de France, IX série, IV (1969), pp. 161-194. On trouvera une étude monographique utile par S. MORALEJO ALVAREZ, Pour l'interprétation iconographique du Portail de l'Agneau à Saint-Isidore de Léon: les signes du zodiaque, in Les Cahiers de Saint-Michel de Cuxa, VIII (1977), pp. 137-173. Pour une époque légèrement plus tardive, et en général : H. BOBER, The Zodiacal Miniature of the Très riches heures of the Duke of Berry. Its Sources and Meaning, in Journal of the Warburg and Courtauld Institute, XI (1948), pp. 1-34; W. HÜBNER, Zodiacus Christianus. Jûdisch-christliche Adaptationen des Tierkreises von der Antike bis zur Gegenwart, Kônigstein,1983 (Beiträge zur Klassischen Philologie, 144).

${ }^{49}$ Dans ce sens je m'éloigne de l'interprétation proposée par P. K. KLEIN, The borders of the Bayeux Tapestry, cit.

${ }^{50}$ M. CAMILLE, Image on the Edge. The Margins of Medieval Art, Londres, 1992; J. WIRTH, Les marges a drôleries des manuscrits gothiques (1250-1350), Genève, 2008.

${ }^{51}$ B. KIILERICH, Ducks, Dolphins and Portrait Medallions : Framing the Achilles Mosaic at Pedrosa de la Vega (Palencia), in Acta ad archaeologiam et artium pertinentia, XV (2001), pp. 245-267.

${ }^{52}$ L. MARIS, The Frame of Painting or the Semiotic Functions of Boundaries in the representative Process, in: S. CHATMAN, U. ECO, J.-M. KLINKENBERG (eds.), A Semiotic Landscape, Den Haag, 1979, pp. 777-782 ; J.-C. LEBENSZTEJN, Framing Classical Space, in Art Journal, 47 (1988), pp. 37-41; P. DURO (ed.), The Rhetoric of the Frame. Essays on the Boundaries of the Artwork, Cambridge, 1996; B. KILLERICH, Frames and Parergonality, in Journal of Aesthetics and Art Criticism, 59 (2001), pp. 320-323

${ }^{53}$ W. GEORGE, Animals and Maps, Londres, 1969; I. GERHARD, Zoologie médiévale: préocupations et procédés, in : Methoden im Wissenschaftund Kunst des Mittelalters, Berlin, 1970 (Miscellanea Mediaevalia, 7), pp. 231-248.

* This work has been supported by the Croatian Science foundation under the project 6095 Croatian Medieval Heritage in European Context: Mobility of Artists and Transfer of Forms, Functions and Ideas (CROMART)
} 


\section{KROTITEU MEDVJEDA S DONJE BORDURE TAPISERIJE IZ BAYEUXA: ZA NOVU INTERPRETACIJU}

Tapiserija iz Bayeuxa prikazuje invaziju Engleske, godine 1066., kad je Vilijam Osvajač porazio Haroldovu englesku vojsku u bitci kod Hastingsa. Njezin bogati sadržaj oslikava kolektivni imaginarij srednjeg vijeka, bivajući ujedno ključnim izvorom informacija za vizualizaciju svakodnevnog života u 11. i prvoj polovini 12. stoljeća. Tapiserija je uokvirena s dvije bordure koje prikazuju fantastične životinje, zvijeri ili basne, poput one o vrani i lisici, kao i ratnike koji su dio šire fabulacije u glavnom okviru. U ovom se tekstu nastoji identificirati jednu od scena u donjoj borduri, onu u kojoj čovjek trenira i pokazuje medvjeda.

Ključne riječi: Bayeux, tapiserija, kroćenje životinja, medvjed, granice, ikonografija 